$12-31-2016$

\title{
The Impact of SHA-1 File Hash Collisions On Digital Forensic Imaging: A Follow-up Experiment
}

Gary C. Kessler

Embry Riddle Aeronautical University - Daytona Beach

Follow this and additional works at: https://commons.erau.edu/jdfsl

Part of the Computer Engineering Commons, Computer Law Commons, Electrical and Computer Engineering Commons, Forensic Science and Technology Commons, and the Information Security Commons

\section{Recommended Citation}

Kessler, Gary C. (2016) "The Impact of SHA-1 File Hash Collisions On Digital Forensic Imaging: A Followup Experiment," Journal of Digital Forensics, Security and Law. Vol. 11 , Article 10.

DOI: https://doi.org/10.15394/jdfsl.2016.1433

Available at: https://commons.erau.edu/jdfsl/vol11/iss4/10

This Article is brought to you for free and open access by

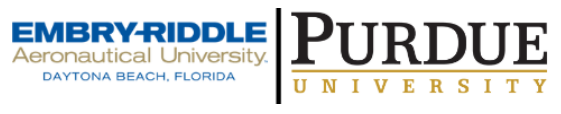
the Journals at Scholarly Commons. It has been accepted for inclusion in Journal of Digital Forensics, Security and Law by an authorized administrator of Scholarly Commons. For more information, please contact commons@erau.edu.

(c)ADFSL 


\title{
THE IMPACT OF SHA-1 FUE HASH COLLISIONS \\ ON DIGITAL FORENSIC IMAGING: A FOLLOW-UP EXPERIMENT
}

\author{
Gary C. Kessler \\ Embry-Riddle Aeronautical University \\ Daytona Beach, Florida \\ 386-226-7947 \\ gary.kessler@erau.edu
}

\begin{abstract}
A previous paper described an experiment showing that Message Digest 5 (MD5) hash collisions of files have no impact on integrity verification in the forensic imaging process. This paper describes a similar experiment applied when two files have a Secure Hash Algorithm (SHA-1) collision.
\end{abstract}

Keywords: SHA-1 hash collisions, forensic imaging, computer forensics, digital forensics

\section{INTRODUCTION}

An earlier paper (Kessler, 2017) discussed the impact on the hash value of two disk images that contain the same set of files except for one -- one file that has the same Message Digest 5 (MD5) hash value as another file of the same size but different content. That paper showed that the resulting disk image hash values were, in fact, different even though all of the component files and spaces on the disk had the same hash.

That paper was specific to MD5 hash collisions. As it was coming to press, Stevens, Bursztein, Karpman, Albertini, and Markov (2017) announced a SHA-1 hash collision between two files of the same size with different content.

This paper will use the same methodology as the earlier paper to address the impact of SHA-1 hash collisions on validating the results of the computer forensics imaging process.
Section 2 will state the research question. Section 3 will describe the experimental framework with which to test the research hypothesis, followed by test results in Section 4. Section 5 will offer conclusions.

\section{RESEARCH QUESTION}

The earlier paper (Kessler, 2017) described a scenario that can be summarized as follows: Suppose we have two files, A and B, that have different content but are the same size and have the same SHA-1 hash value. What is the effect on the hash value of two disk images that differ only in that one disk contains File $\mathrm{A}$ and the other disk contains File B (where Files A and B occupy the same location on the two disk images)? SHA-1 is described in Eastlake and Jones (2001) and NIST (2015).

The research question is to test the following null hypothesis $\left(\mathrm{H}_{0}\right)$ as follows:

- The resultant two disk images will have the same hash value. 
The alternative hypothesis $\left(\mathrm{H}_{1}\right)$ is as follows:

- The resultant two disk images will have different hash values.

\section{EXPERIMENTAL SETUP}

To address the research questions, two files were needed that were the same size, had the same SHA-1 hash, and had different content. Centrum Wiskunde \& Informatica (2017) provides such a pair of 422,435-byte files, called shattered-1.pdf and shattered-2.pdf (Figure 1).

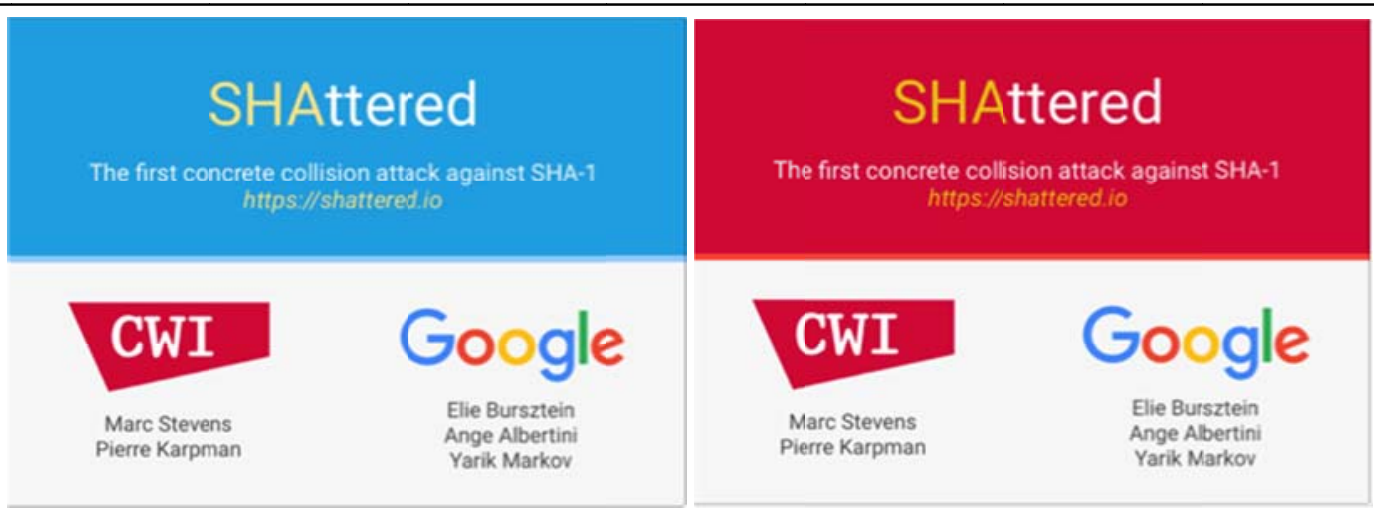

Figure 1. shattered-1.pdf (left) and shattered-2.pdf (right).

Examined in a hex editor, these files are found to be different in 92 nibbles (in 62 bytes), all within a single 128-byte block starting at byte offset 0x00C0; the differences are indicated by the bolded nibbles below:

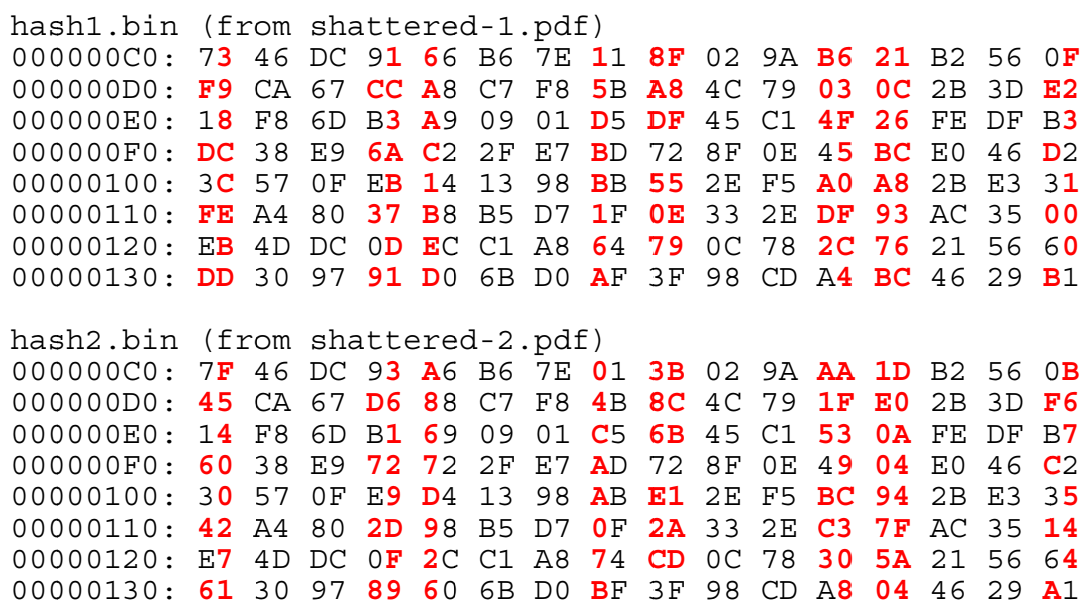

The $f c$ (file compare) command confirms these differences. Details of this comparison, including the 150 bits that differ, can be found in Appendix 1.

As shown below, the two files have the same 160-bit SHA-1 hash, although their 128- bit MD5 hash values differ. This confirms that the contents of the two files are actually different and that there is a bona fide SHA-1 hash collision:

File: shattered-1.pdf

MD5 EE4AA52B139D925F8D8884402B0A750C

SHA 38762CF7F55934B34D179AE6A4C80CADCCBB7F0A 
File: shattered-2.pdf

MD5 5BD9D8CABC46041579A311230539B8D1

SHA 38762CF7F55934B34D179AE6A4C80CADCCBB7FOA

A $32 \mathrm{MB}$ thumb drive was used as the test medium. Using Windows 10, the thumb drive was formatted using the format e: /v:SHATEST /p:1 command. This initialized a FAT16 partition where the data area was overwritten with zeroes. The contents of the thumb drive were verified using the WinHex (v17.5) hex editor. Finally, a set of seven files were copied -- six arbitrary files plus hash1.pdf (containing the contents of shattered-1.pdf) -- to the thumb drive. The file list and hash values were:

File: 100 0230.JPG

MD5 097D23̄B541E4F58F03C57D410C3E3AD5

SHA EB916AF75CB5B5BB145F7C11DF17FEC2B04B4395

File: Charts Navigation.pdf

MD5 4942439FĀ574809EEAFFF72989FE4276

SHA 6DF61583B57FE4832AD5929E14AFA10638836FA9

File: diveboat.jpg

MD5 91700649FD62204C3675A045142424E8

SHA B043E115E14C9EA3 870D2 08526EEF300D4F4CCEC

File: hash1.pdf

MD5 EE4AA52B139D925F8D8884402B0A750C

SHA 38762CF7F55934B34D179AE6A4C80CADCCBB7F0A

File: IMG 1425.JPG

MD5 CB8FE970560AA6184ED1BC2EEC887681

SHA 8A37616C53CD53B1281B32889A07E29EAC99B09B

File: in $5615551872 . \mathrm{flv}$

MD5 27DE3209E3B68414A7429E4104C22185

SHA $40 \mathrm{E} 6 \mathrm{AD} 4$ 8C728C4FF916E354B962FBA4B5C7C77A6

File: PICT0131 GCK.JPG

MD5 A9ABC3E926 $\overline{\mathrm{F}} 93 \mathrm{~A} 03 \mathrm{D} 4844323 \mathrm{~B} 21 \mathrm{C} 513 \mathrm{D}$

SHA C7FD4F3B8F743BF6202E6C57CC621A0EE6F5C6B5

\section{TESTS AND RESULTS}

Four tests were conducted on the media described above. The results described in this section are summarized in Table 1.

In Test \#S1, the thumb drive was imaged using FTK Imager (v3.1.3.2). The purpose of this test was merely to prepare a baseline disk image and set of hash values. The image verification SHA-1 hash of the thumb drive was

0a7c8c48793c0742ae37b9d5b4877ef7700b 9b18 and the complete FTK Imager report can be found in Appendix 2. The image was examined with FTK (v1.81.6) and the file listing for hash1.pdf showed the expected MD5 and SHA-1 hash values for the shattered-1.pdf file (as shown in Section 3).

For Test \#S2, the thumb drive was mounted with WinHex and the contents of hash1.bin were copied over the 128-byte "collision block" of hash1.pdf on the thumb drive (i.e., the 128 bytes starting at offset 0x8490C0 on the image). The purpose of this test was to confirm that overwriting data in this way was possible and reliable. Note that it was not necessary to change anything else on the thumb drive since the two files were the same size; no changes were necessary to the FAT table entries or to the directory name, address, or file size. The thumb drive was then re-imaged. The image verification SHA-1 hash was

\section{0a7c8c48793c0742ae37b9d5b4877ef7700b}

$9 \mathrm{~b} 18$-- the same as in Test \#S1. This result confirms that overwriting data in this way is an adequate process and changes nothing else on the drive. A portion of the FTK Imager report can be found in Appendix 3. The FTK file listing showed that hash1.pdf had the expected MD5 and SHA-1 hash values for the shattered-1.pdf file.

For Test $\#$ S3, the thumb drive was mounted in WinHex and the contents of hash2.bin were copied over the 128-byte "collision block" where hash1.pdf resided on the thumb drive, thus creating the shattered2.pdf file. This test was really the crux of the hypothesis experiment since hash1.pdf now contained the "hash-equivalent, contentdifferent" file. The thumb drive was re-imaged, yielding an image verification SHA-1 hash of a00b80e17de1677d34d21c6e53ff9e0603ead be6 -- different than Tests \#S1 and \#S2. A portion of the FTK Imager report can be found in Appendix 4. The FTK file listing showed 
that hash1.pdf had the expected MD5 and SHA-1 hash values for the shattered-2.pdf file.

For Test \#S4, the thumb drive was mounted with WinHex and the contents of hash1.bin were copied back over the "collision block" where hash1.pdf resided on the thumb drive, now recreating the shattered-1.pdf file. The purpose of this test was to restore the drive to its original state and confirm that Test \#S3 changed nothing more than the 128 bytes where the test data resided. The image verification SHA-1 hash was 0a7c8c48793c0742ae37b9d5b4877ef7700b $9 \mathrm{~b} 18$-- the same as in Tests \#S1 and \#S2. This result confirms that Test \#S4 had restored the disk to its initial state and that Test \#S3 changed nothing more than the file data. A portion of the FTK Imager report can be found in Appendix 5. The FTK file listing showed that hash1.pdf had the expected MD5 and SHA-1 hash values for the shattered-1.pdf file.

\section{CONCLUSIONS}

The image verification SHA-1 hashes in Tests \#S1, \#S2, and \#S4 -- images that each held the shattered-1.pdf (hash1.bin) content -- had the same value, whereas the image verification SHA-1 hash value in Test \#S3 -- when the image held the shattered-2.pdf (hash2.bin) content -- was different from the other tests. The fact that Tests \#S1, \#S2, and \#S4 had the same hash proved that the test process worked as desired; the fact that Test \#S3 had a different result shows that the hash value of the imaged drive depends upon the actual bit content of the entire drive. Since the hash values of the two images are not the same, the null hypothesis $\left(\mathrm{H}_{0}\right)$ is disproven and the alternate hypothesis $\left(\mathrm{H}_{1}\right)$ is proven.

Table 1.

Summary of the four tests and the results.

\begin{tabular}{|c|c|}
\hline Description of Test & Image SHA-1 Hash Value \\
\hline \#S1 - Drive with shattered-1.pdf & 0a7c8c48793c0742ae37b9d5b4877ef7700b9b18 \\
\hline $\begin{array}{l}\text { \#S2 - Overwrite bytes 0x8490C0-0x84913F } \\
\text { hash1.bin data (shattered-1.pdf) }\end{array}$ & 0a7c8c48793c0742ae37b9d5b4877ef7700b9b18 \\
\hline $\begin{array}{l}\text { \#S3 - Overwrite bytes 0x8490C0-0x84913F } \\
\text { hash2.bin data (shattered-2.pdf) }\end{array}$ & $17 \mathrm{de} 1677 \mathrm{~d}$ \\
\hline $\begin{array}{l}\text { \#S4 - Overwrite bytes 0x8490C0-0x84913F } \\
\text { hash1.bin data (shattered-1.pdf) }\end{array}$ & 0a7c8c48793c0742ae37b9d5b4877ef7700b9b18 \\
\hline
\end{tabular}

As in the prior paper, disproving the null hypothesis is the expected result because the hash value of a disk image is based upon the bit contents of the disk rather than the hashes of the individual files -- including file system structures and unallocated space -- that compose the disk contents. Thus, even if all of the file hashes on two disks are the same, the disk image hashes will be different if the contents of the files are different. Given this result, the scenario described in Section 2 cannot be realized.
It is hoped that this result will lay the concern about file hash collisions to rest as they apply to digital forensic imaging. As long as both individual files and the entire image are hashed, the theoretical occurrence of individual file collisions is not a factor in confirming the evidentiary integrity of a forensic copy.

This said, the fact that SHA-1 collision can be forced is significant. Although the SHA-1 standard was deprecated in 2013, it is still in wide use. 
As noted in the prior paper, the MD5 hash values are different for the shattered-1.pdf and shattered-2.pdf files, although the SHA-1 hash value is the same. Since the MD5 and SHA-1 algorithms are different, the manipulation that can create an MD5 collision cannot create a SHA-1 collision -- indeed, note the complexity of the SHA-1 collision compared to the relative simplicity of the mD5 collision. To date, no one has yet shown a practical method with which to cause both an MD5 and SHA-1 collision in the same file.

\section{NOTE}

All FTK Imager reports, FTK reports, and ancillary files are available for examination at http://www.garykessler.net/gck/sha_test.zip.

\section{AUTHOR BIOGRAPHY}

Gary C. Kessler, Ph.D., is a professor of cybersecurity and chair of the Security Studies \& International Affairs Department at EmbryRiddle Aeronautical University in Daytona Beach, Florida. He is a Certified Computer Examiner (CCE), Certified Cyber Forensics Professional (CCFP), and Certified Information Systems Security Professional (CISSP), and a member of the Hawaii and North Florida Internet Crimes Against Children (ICAC) Task Force. Additional information can be found at http://www.garykessler.net. 


\section{REFERENCES}

Centrum Wiskunde \& Informatica (CWI). (2017). Shattered. Retrieved from https://shattered.it/

Eastlake, D., 3rd, \& Jones, P. (2001, September). US Secure Hash Algorithm 1 (SHA1). Requests for Comments (RFC) 3174. Retrieved from https://www.rfceditor.org/rfc/rfc3174.txt

Kessler, G.C. (2017). The Impact of MD5 File Hash Collisions on Digital Forensic Imaging. Journal of Digital Forensics, Security \& Law, Vol. 11: No. 3, pp. 129140.

National Institute of Standards and Technology (NIST). (2015, August). Secure Hash Standard (SHS). Federal Information Processing Standards Publication FIPS PUB 180-4. Retrieved from http://csrc.nist.gov/publications/fips/fips1 80-4/fips-180-4.pdf

Stevens, M., Bursztein, E., Karpman, P., Albertini, A., \& Markov, Y. (2017). The first collision for full SHA-1. Retrieved from https://shattered.it/static/shattered.pdf 


\section{APPENDICES}

\section{Appendix 1: Comparison of 128-Byte Difference of the Two Files}

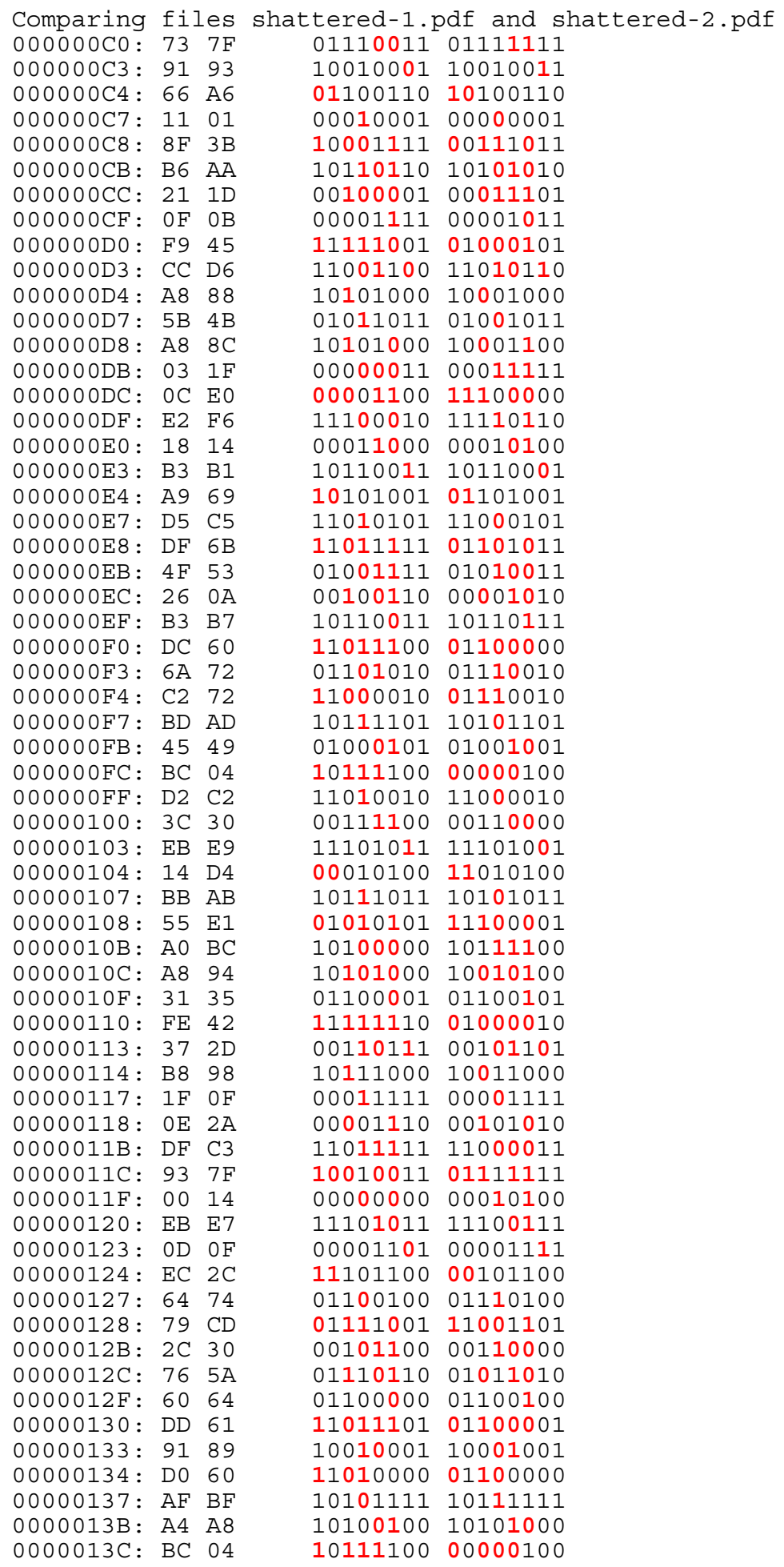

(C) 2016 ADFSL 
0000013F: B1 A1 1011000110100001

Although beyond the scope of this paper, a pattern emerges when looking at the bytes bit-by-bit. The following table shows the values of the 128-byte "difference" block when the two files are ExclusivelyORed (XOR) together; a 0 indicates bits that are the same in the two blocks and a 1 indicates bits that are flipped:

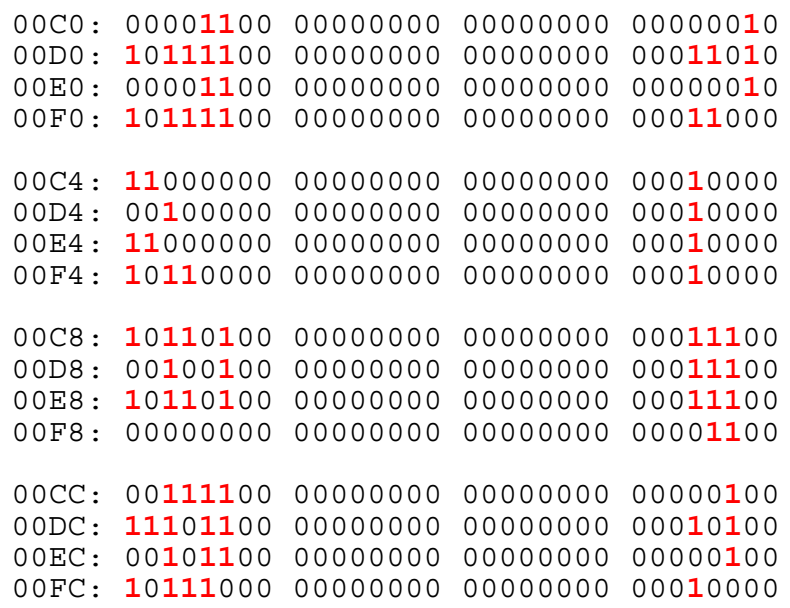

The table above only shows the portion of the block from offset 0x00C0-00FF; the block from offset 0x0100-0x013F exhibits the same pattern.

In summary, 62 bytes of the 128-byte block (48.4\%) are different, including 92 of the 256 nibbles (35.9\%) and 150 of the 1,024 bits $(14.6 \%)$.

\section{Appendix 2: FTK Imager report for Test \#S1}

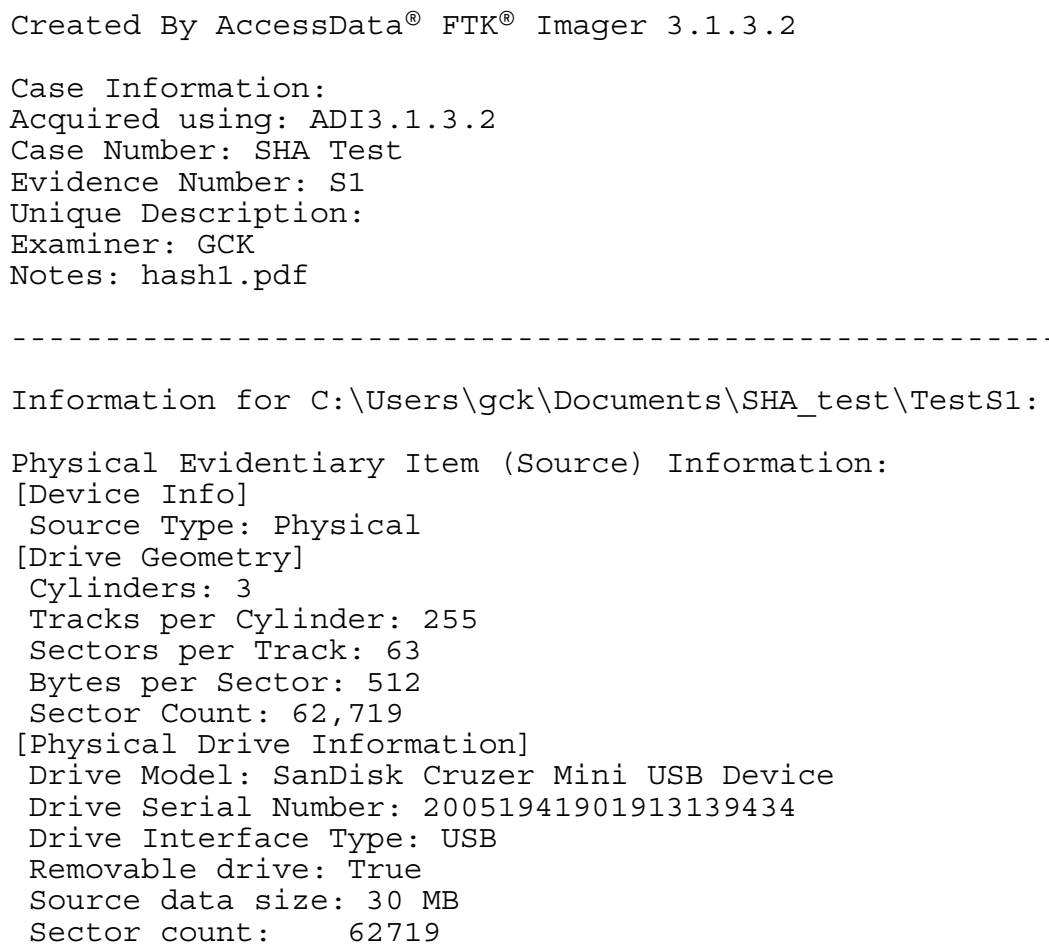




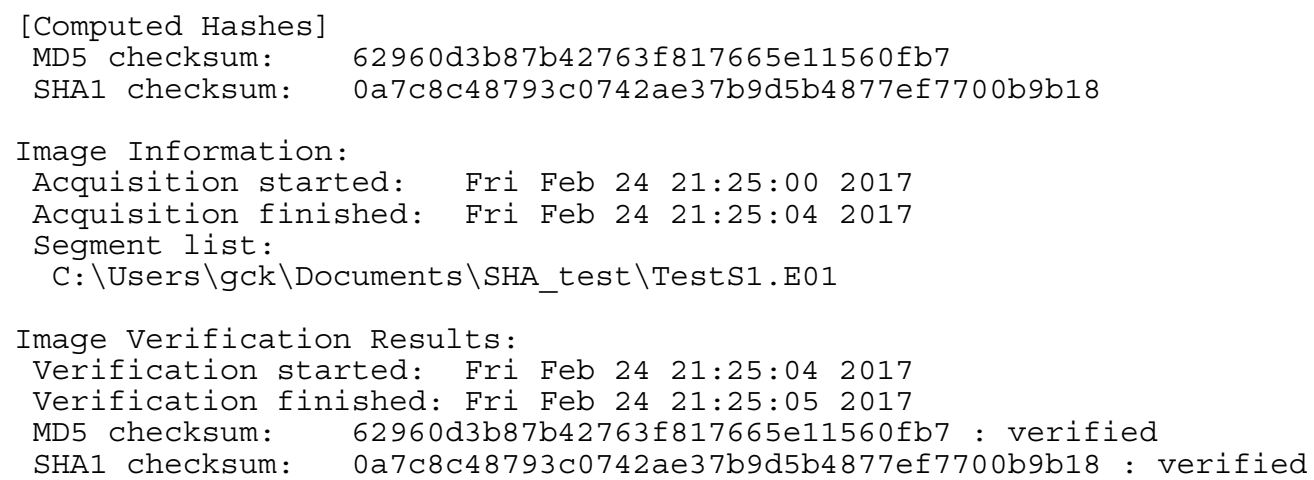

\section{Appendix 3: FTK Imager report (partial) for Test \#S2}

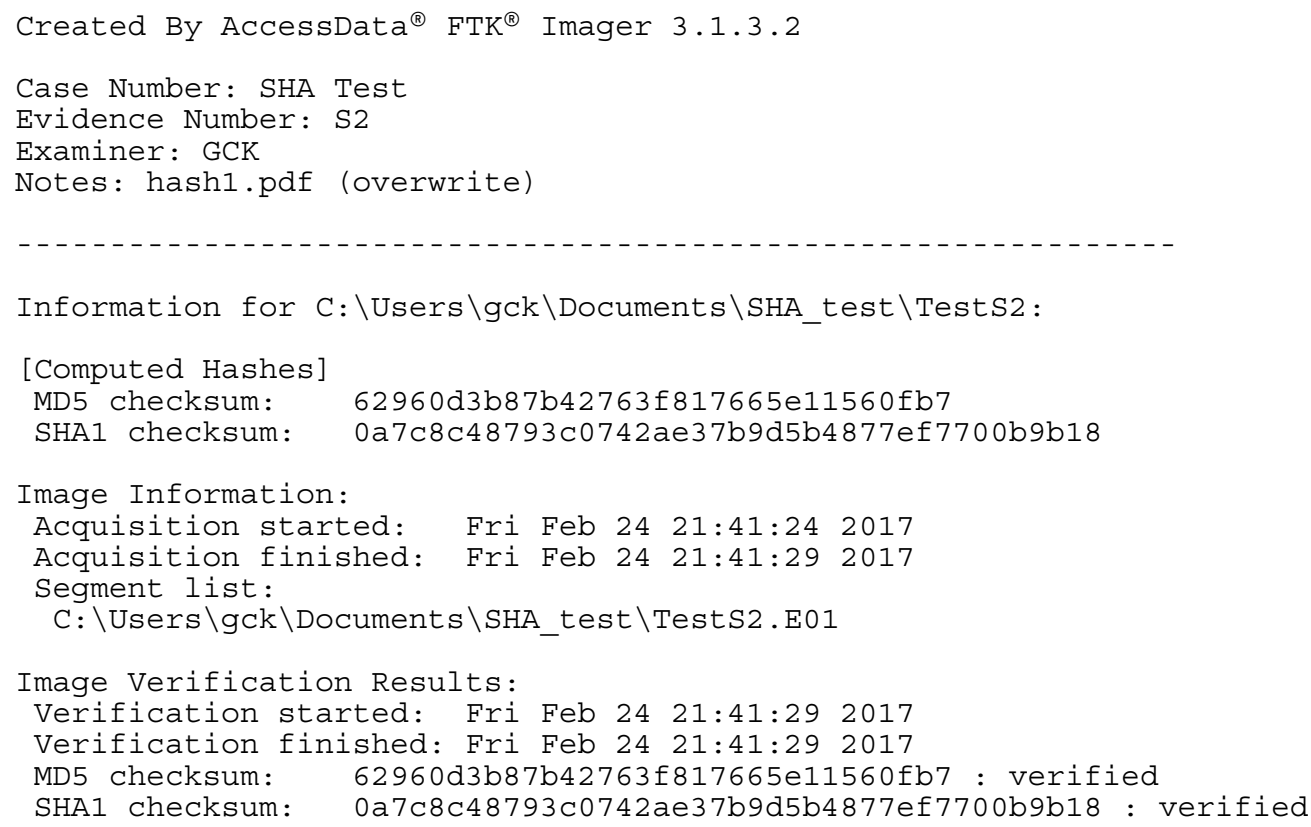

\section{Appendix 4: FTK Imager report (partial) for Test \#S3}

Created By AccessData ${ }^{\circledR}$ FTK $^{\circledR}$ Imager 3.1.3.2

Case Information:

Case Number: SHA Test

Evidence Number: S3

Examiner: GCK

Notes: hash2.pdf overwrite

Information for $\mathrm{C}: \backslash$ Users $\backslash$ gck $\backslash$ Documents $\backslash$ SHA_test $\backslash$ Tests3:

[Computed Hashes]

MD5 checksum: $5704 \mathrm{f9b} 18354 \mathrm{cc} 804 \mathrm{c} 08 \mathrm{~b} 3836 \mathrm{e} 87 \mathrm{~d} 43 \mathrm{f}$

SHA1 checksum: a00b80e17de1677d34d21c6e53ff9e0603eadbe6

Image Information:

Acquisition started: Fri Feb 24 21:52:56 2017

Acquisition finished: Fri Feb 24 21:53:00 2017

Segment list:

C: \Users \gck \Documents $\backslash$ SHA_test $\backslash$ TestS3.E01 


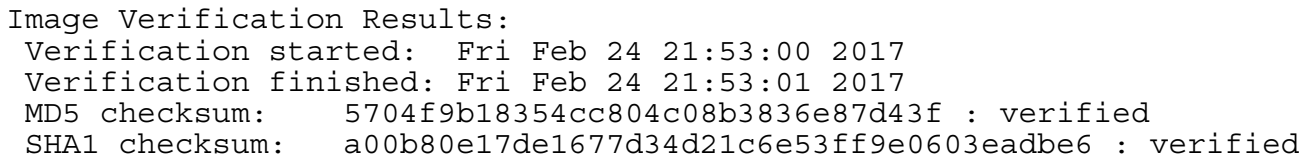

\section{Appendix 5: FTK Imager report (partial) for Test \#S4}

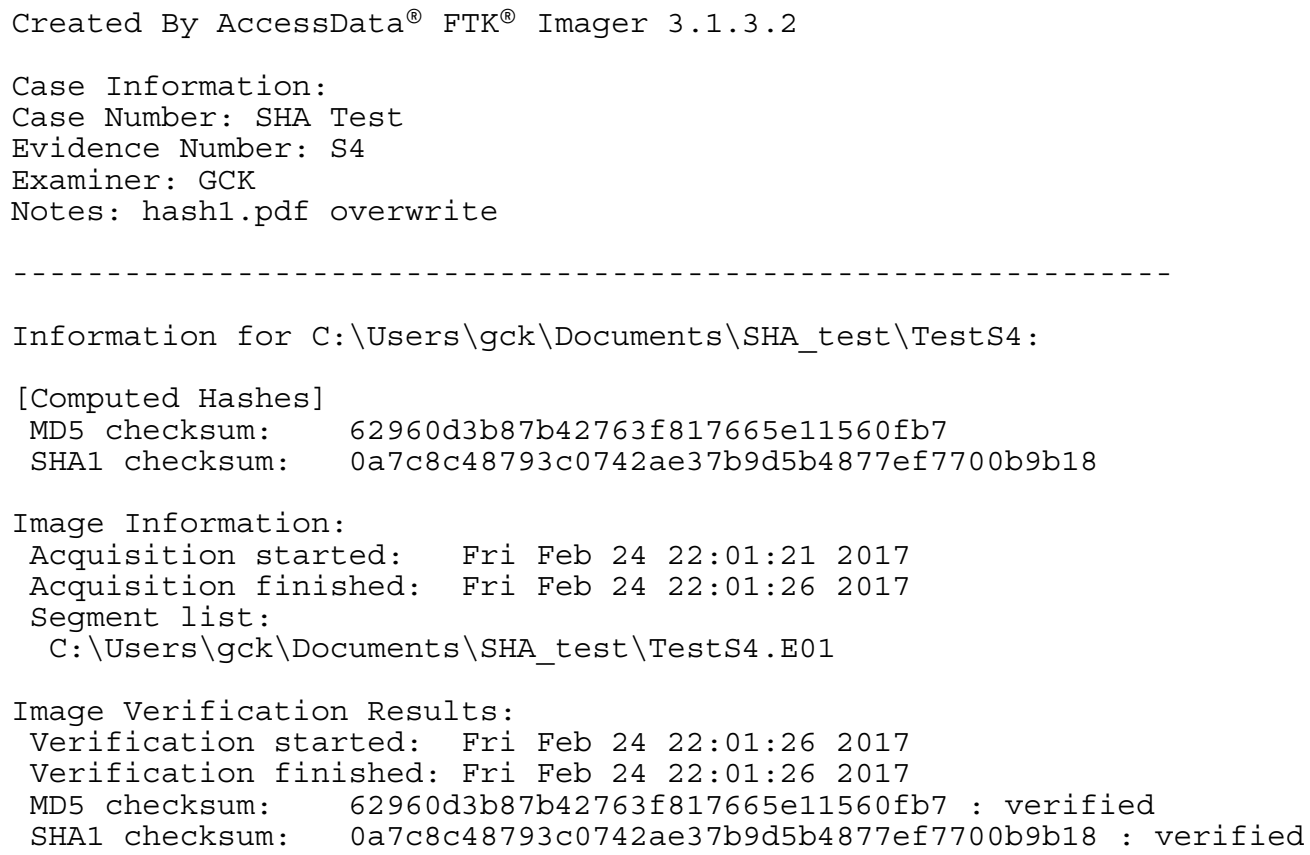

